Revista Brasil. Bot., V.34, n.3, p.411-422, jul.-set. 2011

\title{
Composição florística das espécies vasculares e caráter sucessional da flora arbórea de um fragmento de Floresta Estacional Semidecidual no Sul do Brasil ${ }^{1}$
}

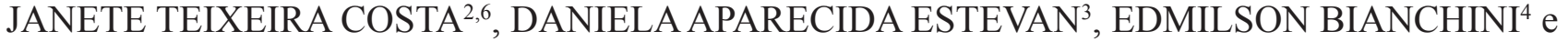 \\ INÊS CRISTINA DE BATISTA FONSECA ${ }^{5}$
}

(recebido: 17 de junho de 2010; aceito: 11 de agosto de 2011)

\begin{abstract}
Floristic composition of the vascular species and tree flora successional character in a Seasonal Semideciduous Forest remnant in Southern Brazil). The floristic composition of vascular species present in an urban forest remnant in the

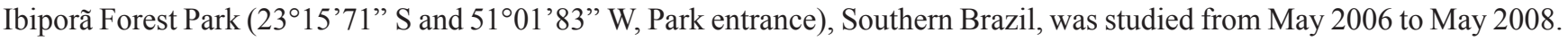
The species were divided in life form and tree species were placed in successional categories. A total of 176 native species, 147 genera and 57 families were found. The majority of species were Angiosperms (98\%) while 2\% were Pteridophyta. The richest families were Fabaceae (19), Bignoniaceae, Malvaceae (nine), Meliaceae and Rubiaceae (eight). The tree species were majority $(53 \%)$, following by herbaceous $(16 \%)$, vines $(14 \%)$, shrubs $(13 \%)$ and epiphyte species $(4 \%)$. The successional characterization of the tree species showed a slight predominance of the late secondary species $(52 \%)$ over species of early succession (45\%), indicating that the vegetation of the PFI is in the intermediate to advanced stage of ecological succession.
\end{abstract}

Key words - ecological succession, forest remnant, richness, successional groups, urban park

RESUMO - (Composição florística das espécies vasculares e caráter sucessional da flora arbórea de um fragmento de Floresta Estacional Semidecidual no Sul do Brasil). Foi realizado o levantamento florístico de espécies vasculares em remanescente urbano de Floresta Estacional Semidecidual no Parque Florestal de Ibiporã $\left(23^{\circ} 15^{\prime} 71^{\prime}\right.$ ' S e $51^{\circ} 01^{\prime} 83^{\prime}$ " W, entrada do Parque), entre maio de 2006 e maio de 2008. As espécies foram classificadas quanto às formas de vida e, as espécies arbóreas, em categorias sucessionais. Foram amostradas 176 espécies nativas, distribuídas em 147 gêneros e 57 famílias. Das espécies, 98\% eram Angiospermas e 2\% Pteridófitas. As famílias com maior riqueza em espécies foram Fabaceae (19), Bignoniaceae, Malvaceae (nove), Meliaceae e Rubiaceae (oito). Quanto às formas de vida, 53\% eram espécies arbóreas, 16\% herbáceas, 14\% lianas, $13 \%$ arbustivas e $4 \%$ epífitas. A classificação das espécies arbóreas em grupos ecológicos indicou ligeira predominância de espécies dos estádios finais (52\%) sobre as espécies dos estádios iniciais da sucessão (45\%), indicando que a vegetação do PFI encontra-se em estádio intermediário a avançado da sucessão ecológica.

Palavras-chave - grupos sucessionais, parque urbano, remanescente florestal, riqueza, sucessão ecológica

\section{Introdução}

A fragmentação das florestas reduz a área de cobertura total e promove alterações no ambiente florestal, podendo resultar na extinção imediata de algumas espécies e, ao longo do tempo, expõe os organismos sobreviventes aos efeitos do ambiente alterado, o que se tem denominado de efeito de borda

Trabalho desenvolvido no Programa de Pós-Graduação em Agronomia, Universidade Estadual de Londrina, Londrina, PR, Brasil.

2. Instituto Ambiental do Paraná, Caixa Postal 600, 86051-990 Londrina, PR, Brasil.

3. Centro Universitário Filadélfia - UNIFIL. Avenida Juscelino Kubitschek, 1626, 86020-000 Londrina, PR, Brasil.

4. Universidade Estadual de Londrina, Departamento de Biologia Animal e Vegetal, Caixa Postal 6001, 86051-990 Londrina, PR, Brasil.

5. Universidade Estadual de Londrina, Departamento de Agronomia, Caixa Postal 6001, 86051-990 Londrina, PR, Brasil.

6. Autor para correspondência: janetecosta@iap.pr.gov.br
(Murcia 1995). A resposta das espécies é variável, porém alterações na sobrevivência, na estrutura e na distribuição das espécies na comunidade contribuem para a perda da diversidade biológica, tanto diretamente pela redução do estoque de genes e de espécies, como indiretamente, em consequência da quebra das interações ecológicas e da estabilidade dos ecossistemas (Futuyma 1996). O conhecimento da flora e da fauna constitui o ponto de partida para o acompanhamento das alterações temporais que podem orientar ações de manejo das unidades de conservação.

Na região norte do Estado do Paraná, o processo de ocupação com a lavoura cafeeira a partir da década de 40 gerou um intenso processo de fragmentação da floresta original, restando atualmente vários fragmentos pequenos e isolados (Campos \& Costa Filho 2006). Um desses remanescentes é o Parque Florestal de Ibiporã que foi instituído em área de terras devolutas do Estado do Paraná. Este Parque está inserido na formação Floresta Estacional Semidecidual (FES) (Veloso et al. 1991) que, 
no Brasil, se estende por vários Estados, especialmente São Paulo e Paraná. Em muitos locais, a FES ocorre na forma de disjunções florestais sendo constituídas por diferentes espécies, o que demarca domínios florísticos diferentes (Leitão Filho 1987).

Estudos florísticos e fitossociológicos realizados na FES têm evidenciado que os remanescentes podem apresentar floras muito distintas, mesmo quando geograficamente próximos (Gandolfi et al. 1995). A comparação em riqueza de espécies arbóreas realizada em remanescentes florestais na Bacia do Rio Tibagi, Estado do Paraná (Torezan \& Silveira, 2002) mostrou que ocorre alta taxa de substituição de espécies ( $\beta$ diversidade) em função da heterogeneidade ambiental observada no eixo sul-norte.

Em nível local, fatores bióticos (Kinoshita et al. 2006) e abióticos (Bianchini et al. 2001, Botrel et al. 2002, Carvalho et al. 2005, 2009, KotchetkoffHenriques et al. 2005, Bianchini et al. 2010) interferem na dinâmica das florestas. Além destes, o histórico de perturbação do local e a fase da sucessão secundária em que se encontram as formações vegetais estudadas podem determinar variações na composição das espécies observadas em remanescentes localizados próximos (Durigan et al. 2008).

Visando contribuir para o conhecimento da flora e, adicionalmente, para a dinâmica sucessional das FES, foi realizado o levantamento florístico das espécies vasculares do Parque Florestal de Ibiporã, o enquadramento das espécies segundo a forma de vida e, adicionalmente, as espécies arbóreas foram classificadas em grupos sucessionais, o que permitiu avaliar qualitativamente o estágio de desenvolvimento da vegetação deste remanescente.

\section{Material e métodos}

O fragmento de Floresta Estacional Semidecidual Submontana estudado está inserido no Parque Florestal de Ibiporã (PFI), que integra o Sistema Nacional de Unidades de Conservação - SNUC (MMA 2000). O PFI possui área de 74,05 ha e está localizado na região urbana do Município de Ibiporã, PR $\left(23^{\circ} 15^{\prime} 71^{\prime}\right.$ 'S e $51^{\circ} 01^{\prime} 83^{\prime \prime} \mathrm{W}$, na entrada do Parque). O relevo é suavemente ondulado e a altitude varia de $400 \mathrm{~m}$ a pouco mais que $500 \mathrm{~m}$, com declividade em direção norte e oeste.

O fragmento florestal se apresenta localmente como formação secundária, devido ao intenso processo de extração de madeira ocorrido até a década de 1980 sem, contudo, ter sofrido supressão total de vegetação. Foram descritas 72 espécies arbóreas da flora nativa e exótica, por ocasião da implantação do PFI (Roderjan \& Medeiros 1980).
Posteriormente, foram identificadas 39 espécies exóticas, a maioria delas plantadas na década de 80 , para recuperação de áreas cultivadas em anos anteriores (Costa et al. 2008).

$\mathrm{O}$ clima da região é $\mathrm{Cfa}$, subtropical úmido, mesotérmico, com verões quentes e ocorrência pouco frequente de geadas, segundo classificação de Koeppen (Caviglione et al. 2000). Temperatura média anual de $21,9^{\circ} \mathrm{C}$, sendo julho o mês mais frio (média mínima de $13,0^{\circ} \mathrm{C}$ ) e fevereiro o mês mais quente (média máxima de $30,3^{\circ} \mathrm{C}$ ). A precipitação anual é de $1.507 \mathrm{~mm}$, ocorrendo um período de maior pluviosidade de novembro até fevereiro, com pico em janeiro $(214,1 \mathrm{~mm})$ e um período de menor pluviosidade nos demais meses, com valores mais baixos no mês de agosto $(48,7 \mathrm{~mm})$. Os dados, referentes ao período de 1973 até 2008, foram obtidos na Estação Meteorológica de Ibiporã do Instituto Agronômico do Paraná, que dista, aproximadamente, $2 \mathrm{~km}$ do PFI. Os tipos de solos predominantes são o Latossolo Vermelho Eutroférrico e o Neossolo Litólicos Eutróficos (IAP 2009), ocorrendo afloramento de rocha em áreas ao sul e em áreas centrais do Parque.

A paisagem do entorno do PFI é composta por rodovias, estradas municipais, áreas de agricultura convencional, pedreira e área urbana. $\mathrm{O}$ único corredor de vegetação que liga o PFI a outros remanescentes é a mata ciliar do ribeirão Jacutinga e seu tributário, o Córrego Ipê.

A coleta de material botânico reprodutivo foi realizada durante caminhadas ao longo da trilha principal que percorre o PFI formando uma elipse de $2.600 \mathrm{~m}$ e que abrange toda sua extensão e em pequenas trilhas no interior da mata, de modo a abranger todas as regiões do PFI, assim como o seu entorno. Para algumas espécies que não se reproduziram no período do estudo, a identificação foi baseada em material vegetativo.

As caminhadas foram mensais entre maio de 2006 e maio de 2008, com duração de três a quatro horas. Adicionalmente foram incluídas coletas ocasionais realizadas em 2009 e as coletas realizadas pelos guardas-parque do PFI. Foram feitas, em caminhadas no interior da mata, objetivando registrar a localização preferencial das espécies, seja pela topografia ou pelo histórico de perturbações das diferentes regiões do Parque.

As amostras foram coletadas com o auxílio de tesoura de poda e podão, etiquetadas e encaminhadas ao herbário da Universidade Filadélfia de Londrina (UNIFIL). O material coletado foi herborizado, segundo técnicas usuais (Fidalgo \& Bononi 1989), sendo identificado por comparação com as exsicatas dos Herbários UNIFIL e FUEL, da Universidade Estadual de Londrina e por especialistas. As exsicatas foram depositadas nesses herbários. As fanerógamas foram agrupadas em famílias de acordo com o sistema APG III (2009). A espécies estão de acordo com a lista de espécies da flora do Brasil (http://floradobrasil.jbrj.gov.br) e a grafia do nome das espécies e de seus autores está de acordo com o site www.theplantlist.org.

Com base em Rizzini (1997), as espécies foram classificadas em formas de vida arbórea, arvoreta, arbustiva, herbácea, lianas e epífitas. A espécie foi considerada arbórea 
quando seus indivíduos reprodutivos (adultos) possuíam mais de 4 m de altura, com tronco diferenciado e CAP (circunferência a altura do peito - a 1,30 m do solo) igual ou superior a $0,15 \mathrm{~m}$; arvoreta quando os indivíduos reprodutivos possuíam tronco diferenciado e medidas inferiores àquelas utilizadas para caracterizar os indivíduos arbóreos; arbustivo quando os indivíduos adultos lenhosos ou semilenhosos apresentavam-se ramificados desde a base; herbáceo quando os indivíduos adultos eram herbáceos ou semilenhosos ramificados e com altura de até $30 \mathrm{~cm}$; epífitas, quando os indivíduos se desenvolvem sobre outros vegetais, sem dependência nutricional; lianas, quando os indivíduos herbáceos ou lenhosos e fixados no substrato crescem apoiando-se em outras plantas.

As espécies arbóreas e as arvoretas foram enquadradas como espécies das fases iniciais ou das fases finais da sucessão, de acordo com a literatura (Kageyama \& Castro 1989, Gandolfi et al. 1995, Silva \& Soares-Silva 2000, Nunes et al. 2003, Oliveira Filho et al. 2004) e com observações de campo.

\section{Resultados e discussão}

Riqueza florística e hábito - Foram registradas 57 famílias, 147 gêneros e 176 espécies nativas, das quais 13 foram identificadas somente em nível de gênero e cinco em nível de família. Entre estas, cerca de $2 \%$ são Pteridófitas e 98\% são de Angiospermas, distribuídas entre Magnoliídeas (4\%), Monocotiledôneas (cerca de
10\%) e Eudicotiledôneas (cerca de 85\%) (tabela 1). As 10 famílias com maior riqueza em espécies foram Fabaceae (19), Bignoniaceae, Malvaceae (nove), Meliaceae e Rubiaceae (oito), Solanaceae (sete), Asteraceae e Bromeliaceae (seis), Euphorbiaceae, Malpighiaceae e Myrtaceae (cinco), compreendendo cerca de 50\% de todas as espécies amostradas. Das demais famílias, cerca de 37\% (21) apresentaram apenas uma espécie (tabela 1).

Os gêneros com maior riqueza foram Trichilia e Solanum com quatro espécies e Casearia, cordia, Eugenia, Piper e Tillandsia com três. Cerca de $86 \%$ dos gêneros estão representados por uma única espécie (tabela 1). Os quatro primeiros gêneros relacionados acima são descritos por Dias et al. (2002) entre os de maior riqueza na Bacia do Rio Tibagi.

Quanto à forma de vida, 93 espécies (53\%) são arbóreas, incluindo as arvoretas; 28 (16\%) são herbáceas, 25 (14\%) são lianas, $22(13 \%)$ são arbustivas e oito (4\%) são epífitas (tabela 1). A forma de vida arbórea predominou nas famílias Fabaceae (16 espécies), Meliaceae (oito), Myrtaceae, Malvaceae e Rubiaceae (cinco) que, no conjunto, representam $42 \%$ das espécies arbóreas amostradas (tabela 1). As espécies arbustivas estão presentes principalmente nas famílias Solanaceae e Acanthaceae, com quatro espécies cada (tabela 1).

Tabela 1. Composição florística do Parque Florestal de Ibiporã, PR. FV - Forma de vida $(A v=$ arbóreo; $A t=$ arvoreta; $A b=$ arbustivo; $\mathrm{He}=$ herbáceo; $\mathrm{Li}$ = liana; Ep = epífito); $\mathrm{GE}$ - grupo ecológico das espécies arbóreas (IS = espécies do início da sucessão; FS = espécies do final da sucessão; $\mathrm{NC}=$ não classificada); $\mathrm{NR}=$ Número de registro do material testemunho no herbário da Universidade de Londrina; $\mathrm{S} / \mathrm{R}=$ Material vegetativo testemunho.

Table 1. Floristic composition of the vascular species in the Forest Park at Ibiporã, PR. FV - life form $(A v=$ tree; $A t=$ treelet; $\mathrm{Ab}=$ shrub; $\mathrm{He}=$ her; $\mathrm{Li}=$ vines; $\mathrm{EP}=$ epiphyte) $\mathrm{GE}$ - ecological group of tree (IS species of early succession; $\mathrm{FS}=$ species of late succession; $\mathrm{NC}=$ un-classified); $\mathrm{NR}=$ voucher register's number in the Universidade Estadual de Londrina Herbarium; $\mathrm{S} / \mathrm{R}=$ voucher vegetative.

\begin{tabular}{|c|c|c|c|}
\hline FAMÍLIAS/ESPÉCIES & FV & GE & NR \\
\hline \multicolumn{4}{|l|}{ PTERIDÓFITAS } \\
\hline \multicolumn{4}{|l|}{ POLYPODIACEAE } \\
\hline Microgramma lindbergii (Mett. ex Kuhn) de la Sota & Ep & & G.M.F. 07 \\
\hline Microgramma vacciniifolia (Langsd. \& Fisch.) Copel. & Ep & & G.M.F. 139 \\
\hline \multicolumn{4}{|l|}{ SCHIZAEACEAE } \\
\hline Anemia phyllitidis (L.) Sw. & $\mathrm{He}$ & & D.A.E. 1300 \\
\hline \multicolumn{4}{|l|}{ MAGNOLIÍDEAS } \\
\hline \multicolumn{4}{|l|}{ ANNONACEAE } \\
\hline Annona cacans Warm. & $\mathrm{Av}$ & FS & D.A.E. 1372 \\
\hline \multicolumn{4}{|l|}{ LAURACEAE } \\
\hline Endlicheria paniculata (Spreng.) J.F. Macbr. & $\mathrm{Av}$ & FS & D.A.E. 1338 \\
\hline Nectandra megapotamica (Spreng.) Mez & $\mathrm{Av}$ & FS & G.M.F. 166 \\
\hline \multicolumn{4}{|l|}{ MONIMIACEAE } \\
\hline Mollinedia widgrenii A. DC. & Av & FS & G.M.F. 10 \\
\hline
\end{tabular}


continuação

\begin{tabular}{|c|c|c|c|}
\hline FAMÍLIAS/ESPÉCIES & $\mathrm{FV}$ & GE & NR \\
\hline \multicolumn{4}{|l|}{ PIPERACEAE } \\
\hline Piper aduncum L. & $\mathrm{Ab}$ & & G.M.F. 75 \\
\hline Piper amalago $\mathrm{L}$. & $\mathrm{Ab}$ & & D.A.E. 1312 \\
\hline Piper gaudichaudianum Kunth & $\mathrm{Ab}$ & & D.A.E. 1181 \\
\hline \multicolumn{4}{|l|}{ MONOCOTILEDÔNEAS } \\
\hline \multicolumn{4}{|l|}{ ARACEAE } \\
\hline Philodendron renauxii Reitz & Ep & & G.M.F. 170 \\
\hline \multicolumn{4}{|l|}{ ARECACEAE } \\
\hline Euterpe edulis Mart. & Av & FS & $\mathrm{S} / \mathrm{R}$ \\
\hline Syagrus romanzoffiana (Cham.) Glassman & Av & FS & $\mathrm{S} / \mathrm{R}$ \\
\hline \multicolumn{4}{|l|}{ BROMELIACEAE } \\
\hline Acanthostachys strobilacea (Schult. \& Schult.f.) Klotzsch & Ep & & P.R.G. 14 \\
\hline Aechmea distichantha Lem. & $\mathrm{He}$ & & D.A.E. 1207 \\
\hline Ananas bracteatus (Lindl.) Schult \& Schult. f. & $\mathrm{He}$ & & G.M.F. 14 \\
\hline Tillandsia recurvata (L.) L. & Ep & & J.L.A.S. 03 \\
\hline Tillandsia tricholepis Baker & Ep & & D.A.E. 1290 \\
\hline Tillandsia sp. & Ep & & \\
\hline \multicolumn{4}{|l|}{ COMMELINACEAE } \\
\hline Dichorisandra thyrsiflora J.C. Mikan & $\mathrm{He}$ & & D.A.E. 1182 \\
\hline \multicolumn{4}{|l|}{ CYPERACEAE } \\
\hline Cyperus sp. & $\mathrm{He}$ & & D.A.E. 1182 \\
\hline Pycreus lanceolatus (Poir.) C.B.Clarke & $\mathrm{He}$ & & R.F.R.S.G. 19 \\
\hline \multicolumn{4}{|l|}{ MARANTACEAE } \\
\hline Maranta sp. & $\mathrm{He}$ & & D.A.E. 1299 \\
\hline \multicolumn{4}{|l|}{ ORCHIDACEAE } \\
\hline Oeceoclades maculata (Lindl.) Lindl. & $\mathrm{He}$ & & D.A.E. 1302 \\
\hline Sp1 & $\mathrm{He}$ & & D.A.E. 1296 \\
\hline \multicolumn{4}{|l|}{ POACEAE } \\
\hline Paspalum sp. & $\mathrm{He}$ & & D.A.E. 1180 \\
\hline Setaria sp. & $\mathrm{He}$ & & D.A.E. 1170 \\
\hline $\mathrm{Sp} 2$ & $\mathrm{He}$ & & G.M.F. 71 \\
\hline \multicolumn{4}{|l|}{ SMILACACEAE } \\
\hline Smilax cognata Kunth & $\mathrm{Li}$ & & G.M.F. 42 \\
\hline \multicolumn{4}{|l|}{ EUDICOTILEDÔNEAS } \\
\hline \multicolumn{4}{|l|}{ ACANTHACEAE } \\
\hline Geissomeria pubescens Nees & $\mathrm{Ab}$ & & G.M.F. 84 \\
\hline Justicia brasiliana Roth & $\mathrm{Ab}$ & & D.A.E. 1203 \\
\hline Justicia lythroides (Ness) V.A.W. Graham & $\mathrm{Ab}$ & & G.M.F. 154 \\
\hline Ruellia sp. & $\mathrm{Ab}$ & & D.A.E 1248 \\
\hline \multicolumn{4}{|l|}{ AMARANTHACEAE } \\
\hline Alternanthera tenella Colla & $\mathrm{He}$ & & D.A.E. 1169 \\
\hline Chamissoa acuminata Mart. & $\mathrm{Ab}$ & & D.A.E. 1172 \\
\hline Chamissoa altissima (Jacq.) Kunth & $\mathrm{Li}$ & & G.M.F. 147 \\
\hline Iresine diffusa Humb. \& Bonpl. ex Willd & $\mathrm{He}$ & & D.A.E. 1396 \\
\hline \multicolumn{4}{|l|}{ ANACARDIACEAE } \\
\hline Astronium graveolens Jacq. & Av & FS & G.M.F. 167 \\
\hline Schinus terebinthifolia Raddi & Av & IS & D.A.E. 1245 \\
\hline \multicolumn{4}{|l|}{ APOCYNACEAE } \\
\hline Asclepias curassavica L. & $\mathrm{He}$ & & R.F.R.S.G. 18 \\
\hline Aspidosperma polyneuron Müll. Arg. & Av & FS & G.M.F. 129 \\
\hline Tabernaemontana catharinensis A. DC. & Av & IS & D.A.E. 1328 \\
\hline
\end{tabular}


continuação

\begin{tabular}{|c|c|c|c|}
\hline FAMÍLIAS/ESPÉCIES & FV & GE & NR \\
\hline \multicolumn{4}{|l|}{ ARALIACEAE } \\
\hline Hydrocotyle leucocephala Cham. \& Schltdl. & $\mathrm{He}$ & & D.A.E 1176 \\
\hline \multicolumn{4}{|l|}{ ASTERACEAE } \\
\hline Chaptalia nutans (L.) Polák & $\mathrm{He}$ & & D.A.E. 1293 \\
\hline Mikania micrantha Kunth & $\mathrm{Li}$ & & D.A.E. 1183 \\
\hline Sphagneticola trilobata (L.) Pruski & $\mathrm{He}$ & & D.A.E. 1225 \\
\hline Tridax procumbens (L.) L. & $\mathrm{He}$ & & D.A.E. 1303 \\
\hline Sp3 & $\mathrm{Ab}$ & & R.L.R.C. 3 \\
\hline $\mathrm{Sp} 4$ & $\mathrm{Av}$ & $\mathrm{NC}$ & G.M.F. 164 \\
\hline \multicolumn{4}{|l|}{ BIGNONIACEAE } \\
\hline Amphilophium crucigerum (L.) L. G. Lohmann & $\mathrm{Li}$ & & G.M.F.100 \\
\hline Bignonia sciuripabula (K.Schum.) L. G. Lohmann & $\mathrm{Li}$ & & D.A.E. 1390 \\
\hline Dolichandra unguis-cati (L.) L. G. Lohmann & $\mathrm{Li}$ & & D.A.E. 1284 \\
\hline Fridericia florida (DC.) L. G. Lohmann & $\mathrm{Li}$ & & D.A.E. 1391 \\
\hline Handroanthus chrysotrichus (Mart. ex DC.) Mattos & $\mathrm{Av}$ & IS & G.M.F.105 \\
\hline Handroanthus impetiginosus Mattos & $\mathrm{Av}$ & FS & G.M.F. 169 \\
\hline Jacaranda puberula Cham. & $\mathrm{Av}$ & IS & $\mathrm{S} / \mathrm{R}$ \\
\hline Mansoa difficilis (Cham.) Bureau \& K. Schum. & $\mathrm{Li}$ & & G.M.F. 78 \\
\hline Pyrostegia venusta (Ker Gawl.) Miers & $\mathrm{Li}$ & & G.M.F. 86 \\
\hline \multicolumn{4}{|l|}{ BORAGINACEAE } \\
\hline Cordia ecalyculata Vell. & $\mathrm{Av}$ & IS & D.A.E. 1244 \\
\hline Cordia trichotoma (Vell.) Arráb. ex Steud. & $\mathrm{Av}$ & FS & D.A.E. 1331 \\
\hline Cordia americana (L.) Gottschling \& J. S. Mill. & $\mathrm{Av}$ & FS & G.M.F. 85 \\
\hline Heliotropium indicum L. & $\mathrm{He}$ & & D.A.E. 1286 \\
\hline \multicolumn{4}{|l|}{ BRASSICACEAE } \\
\hline Lepidium virginicum $\mathrm{L}$. & $\mathrm{He}$ & & R.F.R.S.G. 04 \\
\hline \multicolumn{4}{|l|}{ CACTACEAE } \\
\hline Brasiliopuntia brasiliensis (Willd.) A. Berger & $\mathrm{Av}$ & $\mathrm{NC}$ & D.A.E. 1220 \\
\hline Lepismium warmingianum (K. Schum.) Barthlott & Ep & & G.M.F. 38 \\
\hline Pereskia aculeata Mill. & $\mathrm{Li}$ & & D.A.E. 1383 \\
\hline \multicolumn{4}{|l|}{ CAPPARACEAE } \\
\hline Capparis flexuosa (L.) L. & $\mathrm{Av}$ & IS & G.M.F. 495 \\
\hline Capparis sp. & $\mathrm{Ab}$ & & D.A.E. 1193 \\
\hline \multicolumn{4}{|l|}{ CARICACEAE } \\
\hline Jacaratia spinosa (Aubl.) A.DC. & $\mathrm{Av}$ & FS & G.M.F. 114 \\
\hline \multicolumn{4}{|l|}{ CELASTRACEAE } \\
\hline Maytenus ilicifolia Mart. ex Reissek & $\mathrm{Av}$ & IS & D.A.E. 1238 \\
\hline \multicolumn{4}{|l|}{ EUPHORBIACEAE } \\
\hline Actinostemon concolor (Spreng.) Müll Arg. & $\mathrm{Av}$ & FS & D.A.E. 1320 \\
\hline Alchornea triplinervia (Spreng.) Müll. Arg. & $\mathrm{Av}$ & IS & G.M.F. 05 \\
\hline Caryodendron janeirense Müll. Arg. & $\mathrm{Ab}$ & & D.A.E. 1285 \\
\hline Croton floribundus Spreng. & $\mathrm{Av}$ & IS & D.A.E. 1399 \\
\hline Manihot sp. & At & $\mathrm{NC}$ & D.A.E. 1285 \\
\hline \multicolumn{4}{|l|}{ FABACEAE - CAESALPINIOIDEAE } \\
\hline Bauhinia forficata Link & $\mathrm{Av}$ & IS & D.A.E. 1174 \\
\hline Caesalpinia ferrea Mart. var. leiostachya Benth. & $\mathrm{Av}$ & IS & D.A.E. 1295 \\
\hline Cassia leptophylla Vogel & $\mathrm{Av}$ & IS & D.A.E. 1374 \\
\hline Holocalyx balansae Micheli & $\mathrm{Av}$ & FS & D.A.E. 1341 \\
\hline Peltophorum dubium (Spreng.) Taub. & $\mathrm{Av}$ & IS & G.M.F. 77 \\
\hline \multicolumn{4}{|l|}{ FABACEAE - MIMOSOIDEAE } \\
\hline Acacia velutina $\mathrm{DC}$. & $\mathrm{Li}$ & & D.A.E. 1228 \\
\hline
\end{tabular}


continuação

\begin{tabular}{|c|c|c|c|}
\hline FAMÍLIAS/ESPÉCIES & $\mathrm{FV}$ & GE & NR \\
\hline Acacia sp. & $\mathrm{Av}$ & IS & D.A.E. 1179 \\
\hline Calliandra tweedii Benth. & $\mathrm{Ab}$ & & G.M.F. 22 \\
\hline Inga marginata Kunth & $\mathrm{Av}$ & FS & D.A.E. 1249 \\
\hline Inga striata Benth. & $\mathrm{Av}$ & IS & D.A.E. 1392 \\
\hline Parapiptadenia rigida (Benth.) Brenan & $\mathrm{Av}$ & FS & G.M.F. 35 \\
\hline Piptadenia gonoacantha (Mart.) J. F. Macbr. & $\mathrm{Av}$ & IS & D.A.E. 1314 \\
\hline \multicolumn{4}{|l|}{ FABACEAE - PAPILIONOIDEAE } \\
\hline Canavalia brasiliensis Benth. & $\mathrm{Li}$ & & G.M.F. 156 \\
\hline Centrolobium tomentosum Benth. & $\mathrm{Av}$ & IS & \\
\hline Lonchocarpus muehlbergianus Hassl. & $\mathrm{Av}$ & IS & D.A.E. 1348 \\
\hline Machaerium scleroxylon Tul. & $\mathrm{Av}$ & FS & D.A.E. 1398 \\
\hline Machaerium stipitatum (DC.) Vogel & $\mathrm{Av}$ & IS & D.A.E. 1377 \\
\hline Myroxylon peruiferum L.f. & $\mathrm{Av}$ & FS & \\
\hline Poecilanthe parviflora Benth. & $\mathrm{Av}$ & FS & G.M.F. 141 \\
\hline \multicolumn{4}{|l|}{ LAMIACEAE } \\
\hline Aegiphila sellowiana Cham. & $\mathrm{Av}$ & IS & D.A.E. 1218 \\
\hline Salvia splendens Sellow ex Roem. \& Schult. & $\mathrm{He}$ & & R.F.R.S.G. 08 \\
\hline \multicolumn{4}{|l|}{ LECYTHIDACEAE } \\
\hline Cariniana legalis (Mart.) Kuntze & $\mathrm{Av}$ & FS & \\
\hline \multicolumn{4}{|l|}{ MALPIGHIACEAE } \\
\hline Alicia anisopetala (A. Juss.) W. R. Anderson & $\mathrm{Li}$ & & J.R.R. 07 \\
\hline Dicella nucifera Chodat & $\mathrm{Li}$ & & G.M.F. 70 \\
\hline Heteropterys cochleosperma A. Juss. & $\mathrm{Li}$ & & D.A.E. 1378 \\
\hline Heteropterys pauciflora (A. Juss.) A. Juss. & $\mathrm{Li}$ & & D.A.E. 1330 \\
\hline Mascagnia divaricata (Kunth) Nied. & $\mathrm{Li}$ & & G.M.F. 31 \\
\hline \multicolumn{4}{|l|}{ MALVACEAE } \\
\hline Bastardiopsis densiflora (Hook. \& Arn.) Hassl. & $\mathrm{Av}$ & FS & D.A.E. 1397 \\
\hline Ceiba speciosa (A. St.-Hil.) Ravenna & $\mathrm{Av}$ & FS & G.M.F. 82 \\
\hline Guazuma ulmifolia Lam. & $\mathrm{Av}$ & IS & D.A.E. 1321 \\
\hline Heliocarpus americanus $\mathrm{L}$. & $\mathrm{Av}$ & IS & D.A.E. 1335 \\
\hline Luehea divaricata Mart. & $\mathrm{Av}$ & IS & D.A.E. 1329 \\
\hline Pavonia communis A. St.-Hil. & $\mathrm{Ab}$ & & D.A.E. 1294 \\
\hline Sida rhombifolia L. & $\mathrm{He}$ & & R.F.R.S.G. 17 \\
\hline Sida spinosa $\mathrm{L}$. & $\mathrm{He}$ & & D.A.E. 1304 \\
\hline Sp5 & $\mathrm{Ab}$ & & D.A.E. 1201 \\
\hline \multicolumn{4}{|l|}{ MELASTOMATACEAE } \\
\hline Miconia discolor DC. & $\mathrm{Av}$ & IS & D.A.E. 1177 \\
\hline \multicolumn{4}{|l|}{ MELIACEAE } \\
\hline Cabralea canjerana (Vell.) Mart. & $\mathrm{Av}$ & FS & \\
\hline Cedrela fissilis Vell. & $\mathrm{Av}$ & FS & \\
\hline Guarea kunthiana A. Juss. & $\mathrm{Av}$ & FS & L.R.C.R. 01 \\
\hline Guarea macrophylla Vahl & $\mathrm{Av}$ & FS & R.F.R.S.G. 02 \\
\hline Trichilia catigua A. Juss. & $\mathrm{Av}$ & FS & D.A.E. 1189 \\
\hline Trichilia elegans A. Juss. & $\mathrm{Av}$ & FS & D.A.E. 1188 \\
\hline Trichilia pallens C. DC. & $\mathrm{Av}$ & FS & G.M.F. 11 \\
\hline Trichilia pallida Sw. & $\mathrm{Av}$ & FS & D.A.E. 1191 \\
\hline \multicolumn{4}{|l|}{ MORACEAE } \\
\hline Ficus sp. & $\mathrm{Av}$ & IS & \\
\hline Maclura tinctoria (L.) D. Don ex Steud. & $\mathrm{Av}$ & FS & G.M.F. 136 \\
\hline Sorocea bonplandii (Baill.) W. C. Burger, Lanj. \& Weiss. Boer & $\mathrm{Av}$ & FS & G.M.F. 104 \\
\hline \multicolumn{4}{|l|}{ MYRTACEAE } \\
\hline Campomanesia xanthocarpa (Mart.) O.Berg & $\mathrm{Av}$ & FS & G.M.F. 88 \\
\hline
\end{tabular}


continuação

\begin{tabular}{|c|c|c|c|}
\hline FAMÍLIAS/ESPÉCIES & FV & GE & NR \\
\hline Eugenia blastantha (O.Berg) D.Legrand & $\mathrm{Av}$ & FS & G.M.F. 145 \\
\hline Eugenia neoverrucosa Sobral & Av & FS & G.M.F. 73 \\
\hline Eugenia pyriformis Cambess. & Av & FS & D.A.E. 1226 \\
\hline Myrcia multiflora (Lam.) DC. & Av & FS & D.A.E. 1379 \\
\hline \multicolumn{4}{|l|}{ NYCTAGINACEAE } \\
\hline Bougainvillea spectabilis Willd. & Av & FS & G.M.F. 45 \\
\hline Guapira opposita (Vell.) Reitz & Av & IS & D.A.E. 1247 \\
\hline Pisonia aculeata $\mathrm{L}$. & $\mathrm{Li}$ & & D.A.E. 1334 \\
\hline \multicolumn{4}{|l|}{ OXALIDACEAE } \\
\hline Oxalis rhombeo-ovata A. St.-Hil. & $\mathrm{Ab}$ & & D.A.E. 1171 \\
\hline Oxalis triangularis A. St.-Hil. & $\mathrm{He}$ & & D.A.E. 1224 \\
\hline \multicolumn{4}{|l|}{ PHYLLANTHACEAE } \\
\hline Margaritaria nobilis L.f. & Av & FS & \\
\hline \multicolumn{4}{|l|}{ PHYTOLACCACEAE } \\
\hline Gallesia integrifolia (Spreng.) Harms & Av & IS & D.A.E. 1322 \\
\hline Phytolacca dioica L. & Av & IS & R.F.R.S.G. 11 \\
\hline \multicolumn{4}{|l|}{ PICRAMNIACEAE } \\
\hline Picramnia ramiflora Planch. & $\mathrm{Av}$ & FS & D.A.E. 1230 \\
\hline \multicolumn{4}{|l|}{ POLYGONACEAE } \\
\hline Ruprechtia laxiflora Meisn. & Av & IS & D.A.E. 1236 \\
\hline \multicolumn{4}{|l|}{ PRIMULACEAE } \\
\hline Myrsine umbellata Mart. & Av & IS & \\
\hline \multicolumn{4}{|l|}{ RHAMNACEAE } \\
\hline Colubrina glandulosa G.Perkins & Av & IS & J.R.R. 08 \\
\hline Gouania virgata Reissek & $\mathrm{Li}$ & & D.A.E. 1185 \\
\hline \multicolumn{4}{|l|}{ ROSACEAE } \\
\hline Prunus myrtifolia (L.) Urb. & Av & FS & G.M.F. 24 \\
\hline \multicolumn{4}{|l|}{ RUBIACEAE } \\
\hline Alseis floribunda Schott & Av & IS & G.M.F. 103 \\
\hline Chiococca alba (L.) Hitchc. & $\mathrm{Li}$ & & D.A.E. 1283 \\
\hline Coutarea hexandra (Jacq.) K.Schum. & Av & IS & J.R.R. 01 \\
\hline Geophila macropoda (Ruiz \& Pav.) DC. & $\mathrm{He}$ & & D.A.E. 1186 \\
\hline Manettia sp. & $\mathrm{Li}$ & & D.A.E. 1397 \\
\hline Psychotria carthagenensis Jacq. & At & FS & D.A.E. 1317 \\
\hline Randia armata (Sw.) DC. & Av & IS & D.A.E. 1380 \\
\hline Rudgea jasminoides (Cham.) Müll. Arg. & At & FS & G.M.F. 44 \\
\hline \multicolumn{4}{|l|}{ RUTACEAE } \\
\hline Balfourodendron riedelianum (Engl.) Engl. & Av & FS & D.A.E. 1313 \\
\hline Esenbeckia febrifuga (A. St.-Hil) A. Juss. ex Mart. & Av & IS & D.A.E. 1175 \\
\hline Pilocarpus pennatifolius Lem. & Av & FS & G.M.F. 04 \\
\hline \multicolumn{4}{|l|}{ SALICACEAE } \\
\hline Casearia decandra Jacq. & Av & FS & G.M.F. 74/48 \\
\hline Casearia gossypiosperma Briq. & Av & FS & J.R.R. 05 \\
\hline Casearia sylvestris $\mathrm{Sw}$. & Av & IS & \\
\hline \multicolumn{4}{|l|}{ SAPINDACEAE } \\
\hline Allophylus edulis (A. St.-Hil., A. Juss. \& Cambess.) Radlk. & Av & IS & D.A.E. 1342 \\
\hline Cardiospermum grandiflorum $\mathrm{Sw}$. & $\mathrm{Li}$ & & D.A.E. 1340 \\
\hline Paullinia meliifolia Juss. & $\mathrm{Li}$ & & D.A.E. 1237 \\
\hline Serjania sp. & $\mathrm{Li}$ & & G.M.F. 158 \\
\hline \multicolumn{4}{|l|}{ SAPOTACEAE } \\
\hline Chrysophyllum gonocarpum (Mart. \& Eichler ex Miq.) Engl. & Av & FS & G.M.F. 40 \\
\hline
\end{tabular}


continuação

\begin{tabular}{|c|c|c|c|}
\hline FAMÍLIAS/ESPÉCIES & FV & GE & NR \\
\hline \multicolumn{4}{|l|}{ SOLANACEAE } \\
\hline Brunfelsia pauciflora (Cham. \& Schltdl.) Benth. & $\mathrm{Ab}$ & & D.A.E. 1223 \\
\hline Cestrum intermedium Sendtn. & $\mathrm{Av}$ & IS & D.A.E. 1281 \\
\hline Solanum americanum Mill. & $\mathrm{Ab}$ & & G.M.F. 72/137 \\
\hline Solanum argenteum Dunal & At & IS & D.A.E. 1291 \\
\hline Solanum campaniforme Roem. \& Schult. & $\mathrm{Ab}$ & & D.A.E. 1173 \\
\hline Solanum scuticum $\mathrm{M}$. Nee & $\mathrm{Ab}$ & & D.A.E. 1221 \\
\hline Vassobia breviflora (Sendtn.) Hunz. & At & IS & D.A.E. 1216 \\
\hline \multicolumn{4}{|l|}{ TALINACEAE } \\
\hline Talinum paniculatum (Jacq.) Gaertn. & $\mathrm{He}$ & & D.A.E. 1178 \\
\hline \multicolumn{4}{|l|}{ URTICACEAE } \\
\hline Cecropia cf. glaziovii Snethl. & $\mathrm{Av}$ & IS & G.M.F. 98 \\
\hline Pilea nummulariifolia (Sw.) Wedd. & $\mathrm{He}$ & & D.A.E. 1316 \\
\hline \multicolumn{4}{|l|}{ VERBENACEAE } \\
\hline Aloysia virgata (Ruiz \& Pav.) Juss. & $\mathrm{Av}$ & IS & G.M.F. 34 \\
\hline Petrea volubilis $\mathrm{L}$. & $\mathrm{Li}$ & & R.F.R.S.G. 17 \\
\hline Stachytarpheta cayennensis (Rich.)Vahl & $\mathrm{Ab}$ & & R.F.R.S.G. 9 \\
\hline Stachytarpheta sp. & $\mathrm{Ab}$ & & D.A.E. 1287 \\
\hline \multicolumn{4}{|l|}{ VIOLACEAE } \\
\hline Hybanthus bigibbosus (A. St.-Hil.) Hassl. & $\mathrm{Ab}$ & & G.M.F. 144 \\
\hline
\end{tabular}

Com exceção de Meliaceae e Malvaceae, todas as demais famílias estão entre as dez mais ricas, em espécies arbóreas, da Bacia do Rio Tibagi, em 12 estudos realizados em florestas ciliares e em áreas mais elevadas da encosta dos rios desta bacia (Dias et al. 2002). Se for considerada apenas a região do baixo Tibagi, as famílias Fabaceae, Euphorbiaceae, Meliaceae, Moraceae e Rubiaceae são descritas como típicas desta região, sendo destacada a família Meliaceae entre as mais abundantes.

A semelhança entre os grupos de famílias mais importantes amostrado no PFI e nos remanescentes florestais ao longo da Bacia do Rio Tibagi reflete a influência do escudo Atlântico sobre a flora da FES do norte do Paraná (Leitão Filho 1987). Por outro lado, a FES é caracterizada pela predominância de ecótipos advindos da Amazônia e, nas áreas subtropicais, esta floresta possui dominância de gêneros de ampla distribuição no Brasil como: Parapiptadenia, Peltophorum, Cariniana, Lecythis e Astronium, entre outros (Veloso et al. 1991). Com exceção do gênero Lecythis, os demais estão presentes no remanescente do PFI e marcam a fisionomia da floresta, devido ao porte avantajado de seus indivíduos.

A riqueza em espécies arbóreas amostradas no PFI é expressiva quando comparada com o número total de espécies arbóreas (547) registradas para a Bacia do Rio Tibagi (Dias et al. 2002). Soares-Silva \& Barroso
(1992) registraram 127 espécies ha ${ }^{-1}$ em levantamento fitossociológico realizado em um fragmento florestal com histórico de conservação conhecido próximo ao PFI. Este dado corrobora a observação da expressiva riqueza arbórea do PFI considerando o seu histórico. Segundo Torezan \& Silveira (2002), os remanescentes da FES da bacia do baixo Tibagi apresentam índices de riqueza em espécies comparáveis aos das áreas ribeirinhas do Sudeste brasileiro.

Merece destaque a ocorrência de Aspidosperma polyneuron (Apocynaceae) e Balfourodendron riedelianum (Rutaceae) no PFI, estando estas entre as espécies que caracterizam a formação FES submontana (Roderjan et al. 2002) que, porém, em decorrência da sua intensa exploração no passado, compõem a lista oficial de espécies ameaçadas de extinção, do estado do Paraná (Ibama 1992). Além dessas, o PFI abriga a espécie Jacaratia spinosa (Caricaceae), uma espécie em risco de extinção em nível regional, conforme demonstram Trabaquini et al. (2007) em estudo realizado em vários remanescentes da região norte do Paraná.

Entre as 28 espécies herbáceas amostradas no PFI, uma (Anemia phyllitidis) pertence às Pteridófitas, 11 às Monocotiledôneas e 16 às Eudicotiledôneas. Estas espécies estão reunidas em 19 famílias, sendo Asteraceae e Poaceae (três) as de maior riqueza de espécies, apresentando, aproximadamente, $21 \%$ das espécies herbáceas amostradas. 
A riqueza de herbáceas no PFI é mais facilmente observada nos locais abertos por trilhas e na borda do Parque. No interior da floresta, a menor ocorrência de herbáceas pode estar relacionada a menor intensidade de luz, apesar da deciduidade de árvores do dossel, especialmente nos meses de baixa pluviosidade e temperatura. $\mathrm{O}$ número de espécies amostradas neste estudo é comparável ao obtido por Inácio \& Jarenkow (2008), no trecho de floresta estacional semidecidual do Parque Nacional do Turvo, no oeste do Rio Grande do Sul, onde foram encontradas 29 espécies, estando a família Poaceae (cinco) entre as mais ricas. Esses autores demonstraram que a abertura do dossel, ocasionada pela fragmentação da floresta, foipositivamente correlacionada com o aumento da cobertura da sinúsia herbácea terrícola e que o fator deciduidade da vegetação pode não ter influência sobre a sinúsia herbácea. Pesquisas realizadas com amostragens em grande escala (Meira Neto et al. 2005) demonstraram que a umidade, as características nutricionais do solo e as condições de luminosidade no sub-bosque são fatores associados à riqueza do subbosque.

As lianas apresentaram baixa riqueza específica, em relação aos outros hábitos, representando 14\% do total de espécies amostradas. Das 25 espécies de lianas, 56\% pertencem às famílias Bignoniaceae (seis), Malpighiaceae (cinco) e Sapindaceae (três) (tabela 1), que estão entre aquelas de maior riqueza em espécies de lianas em remanescentes da FES na região Sudeste do Brasil (Stranguetti \& Ranga 1998, Resende \& Ranga 2005, Kinoshita et al. 2006, Guaratini et al. 2008, Barros et al. 2009). Entretanto, em FES localizada em regiões subtropicais, Asteraceae foi a família com a maior riqueza de espécies (Durigon \& Waechter 2011).

O número de espécies de lianas encontradas no PFI é inferior ao encontrado em outros estudos, nos quais o número variou entre 35 e 215 espécies (Stranguetti \& Ranga 1998, Resende \& Ranga 2005, Kinoshita et al. 2006, Guaratini et al. 2008, Barros et al. 2009, Durigon \& Waechter 2011). Essa baixa riqueza não era esperada em razão da sazonalidade da região, onde no inverno chove cerca de duas vezes menos que no verão (Bianchini et al. 2003, 2006). No entanto, a presença de lianas no PFI é bastante expressiva, podendo ser facilmente observada nas bordas, nas áreas mais abertas da floresta e nos locais de solos mais rasos. Nos períodos secos, observa-se com mais facilidade as "torres" formadas por árvores cobertas com cipós.

Mesmo causando prejuízos às árvores que as sustentam, as lianas contribuem para a dieta da fauna, principalmente a dos primatas, representando, em média,
$21 \%$ das espécies de plantas utilizadas como alimento pela fauna, além de desempenharem importante papel na dinâmica florestal (Morellato \& Leitão Filho 1998). Em razão da importância ecológica desta forma de vida, estudos adicionais devem ser conduzidos no PFI, buscando entender a baixa riqueza de espécies.

As espécies de epífitas (oito) representaram 4\% do total amostrados, sendo Bromeliaceae (quatro) a mais rica em espécies (tabela 1). Tillandsia foi o único gênero dessa família com mais de uma espécie (três) (tabela 1), o qual é facilmente observado em áreas abertas. Segundo Roderjan et al. (2002), o gênero Philodendron (Araceae), amostrado no PFI, é um dos mais característicos da FES.

A baixa ocorrência de epífitas observada no PFI é esperada em remanescentes da Floresta Estacional Semidecidual, nos quais se tem encontrado um percentual menor que 5\% desse hábito de vida (Stranghetti \& Ranga 1998, Guaratini et al. 2008). Entretanto, em estudos de longa duração realizados nas regiões norte e oeste do Paraná, foram registradas 29 espécies (Dettke et al. 2008) e 32 espécies (Borgo et al. 2002) e as famílias mais frequentes foram Bromeliaceae, Cactaceae e Polypodiaceae (Dettke et al. 2008) e Orchidaceae, Polypodiaceae e Cactaceae (Borgo et al. 2002). Embora a deciduidade dos indivíduos do dossel e as alterações dos ecossistemas florestais decorrentes da fragmentação e das ações antrópicas contribuam para a redução da riqueza de epífitas (Dettke et al. 2008), estudos adicionais devem ser conduzidos abordando especificamente esta forma de vida.

Sucessão ecológica - A distribuição das espécies arbóreas em grupos ecológicos evidencia uma ligeira predominância de espécies de final de sucessão (52\%) sobre as espécies de início de sucessão (45\%) (tabela 1).

As espécies dos estádios finais da sucessão localizam-se predominantemente nas regiões norte e noroeste do PFI. Nestas áreas, a vegetação possui indivíduos arbóreos de grande porte, apresenta dossel mais fechado e indivíduos em diferentes estádios de desenvolvimento das espécies Aspidosperma polyneuron, Holocalyx balansae, Machaerium scleroxylon, Parapiptadenia rigida e Phytolacca dioica. No entanto, indivíduos de Lonchocarpus muehlbergianus, Luehea divaricata e Piptadenia gonoacantha, espécies típicas de clareiras (Kageyama \& Castro 1989), foram observados nesta região, sugerindo a abertura do dossel, no passado.

As espécies dos estádios iniciais da sucessão foram frequentemente observadas nas porções sul e leste do 
PFI, que sofreram perturbações até cerca de 30 anos atrás. Nestas áreas, são comumente observados indivíduos de Alchornea triplinervia, Cecropia cf. glaziovii, Guazuma ulmifolia, Myrsine umbellata, Piptadenia gonoacantha e Peltophorum dubium além de várias espécies exóticas (Costa et al. 2008).

$\mathrm{Na}$ área central do Parque, a observação da vegetação sugere que ela se encontra em estágio mais avançado de sucessão que a região sul (proporção de espécies de final de sucessão e sub-bosque desenvolvido). São frequentes indivíduos de Astronium graveolens, Balfourodendron riedelianum, Cabralea canjerana, Cedrela fissilis, Campomanesia xanthocarpa, Guarea kunthiana, e Syagrus romanzoffiana. Nessa região, na transição das áreas de solo raso para solos profundos, foram observados indivíduos de grande porte de Aspidosperma polyneuron, Cariniana legalis e Jacaratia spinosa, provavelmente indivíduos remanescentes da floresta, anterior à extração seletiva de madeira.

A topografia e o direcionamento dos ventos de sudeste para noroeste, que causam frequentes derrubadas de árvores e quebra de troncos e galhos, são as principais causas de perturbação da vegetação na região central do PFI. A riqueza de espécies de estádios intermediários da sucessão ecológica tem sido relacionada com a ocorrência de pequenas clareiras, as quais são colonizadas predominantemente por espécies não pioneiras (Whitmore 1982 apud Kageyama \& Castro 1989). Segundo Guaratini et al. (2008), a riqueza das espécies dos estádios iniciais da sucessão tem sido descrita como uma particularidade das florestas semideciduais do Estado de São Paulo, decorrente da deciduidade sazonal de espécies do dossel e do histórico de perturbações antrópicas, como a retirada seletiva de madeira.

Agrupamento de espécies - A ocorrência de solo raso pode ser um fator que determina a ocorrência do agrupamento de duas espécies nativas as quais podem ser consideradas raras nesta formação florestal: Ananas bracteatus (abacaxi-nativo) e Brasiliopuntia brasiliensis (arumbeva-açu) formam uma mancha de cerca de um hectare no sub-bosque da floresta na área central do PFI.

Ananas bracteatus é utilizada como planta ornamental, como cerca-viva e na produção de fibras (Carlier et al. 2004) e foi registrada em estudo florístico realizado no Parque Municipal de Itajuru, Muriaé, Minas Gerais (Paula et al. 2008). Brasiliopuntia. brasiliensis é frequentemente cultivada pelo seu peculiar aspecto ornamental, com vistosas flores amarelas e frutos avermelhados. Ocorre naturalmente na Floresta Atlântica, próximo do nível do mar, continuando pela região subtropical até o Paraguai, Uruguai e Argentina, em áreas de temperatura elevada. Foi registrada, na forma silvestre ou naturalizada, em áreas ripárias, de restinga e de capoeira no Estado do Rio de Janeiro, em área ripária e na zona da mata na Bahia, na Reserva Biológica de Sapitanduva, Antonina, Paraná e apenas como planta cultivada em Santa Catarina (Scheinvar 1985).

Não se pode descartar a possibilidade de introdução antrópica destas espécies no PFI, entretanto, não há registro de cultivos ornamentais destas espécies no Paraná. Associações semelhantes destas duas espécies têm sido encontradas em estudos florísticos recentes em outros remanescentes da FES e Floresta Ombrófila Mista, em áreas de solos pouco profundos, na Bacia do Rio Tibagi (D. A. Estevan, comunicação pessoal). Estas ocorrências podem ser indícios de dispersão natural destas espécies a partir da Floresta Atlântica, no caso de $B$. brasiliensis, ou a partir de áreas de cultivo para ambas as espécies.

Os dados obtidos neste estudo mostram a importância da realização de levantamentos florísticos, evidenciado pela registro da associação de Ananas bracteatus e Brasiliopuntia brasiliensis e de espécies em risco de extinção como Aspidosperma polyneuron e Balfourodendron riedelianum. As análises indicam que a vegetação do PFI se encontra em fase intermediária a avançada da sucessão secundária. A capacidade de regeneração da vegetação corrobora a importância deste remanescente da FES como unidade de conservação ambiental de uso restrito em uma região com baixa cobertura florestal como o norte do Estado do Paraná.

Agradecimentos - Os autores agradecem à Diretoria de áreas Protegidas do Instituto Ambiental do Paraná, pela permissão de pesquisa na unidade de conservação sob sua administração; à curadoria do Herbário FUEL, pela disponibilização das exsicatas para consulta; aos vários estudantes do curso de Ciências Biológicas da Universidade Filadélfia de Londrina e da Universidade Estadual de Londrina, que colaboraram com os trabalhos de campo e preparo do material botânico, em especial a Gabriela da Mota Ferreira, Paulo Henrique Koyashiki e Rodrigo Fernando Rafael da Silva; aos guardasparque do PFI, Wilson Barbosa (in memorian) e Osvaldo Silvestre, pelo auxílio na coleta de amostras.

\section{Referências bibliográficas}

APG III. 2009. An update of the Angiosperm Phylogeny Group classification for the orders and families of flowering plants. Botanical Journal of the Linnaean Society 161:105-121. 
BARROS, A.A.M., RIBAS, L.A. \& ARAÚJO, D.S.D. 2009. Trepadeiras do Parque Estadual Serra da Tiririca (Rio de Janeiro, Brasil). Rodriguésia 60:681-694.

BIANCHINI, E., PIMENTA, J.A. \& SANTOS, F.A.M. 2001. Spatial and temporal variation in the canopy cover in a seasonal semideciduous Forest. Brazilian Archives of Biology and Technology 44:269-276.

BIANCHINI, E., PIMENTA, J.A. \& SANTOS, F.A.M. 2006. Fenologia de Chrysophyllum gonocarpum (Mart. \& Eichler) Engl. (Sapotaceae) em floresta semidecídua do Sul do Brasil. Revista Brasileira de Botânica 29:595-602.

BIANCHINI, E., POPOLO, R.S., DIAS, M.C. \& PIMENTA, J.A. 2003. Diversidade e estrutura de espécies arbóreas em área alagável do município de Londrina, sul do Brasil. Acta Botanica Brasilica 17:405-419.

BIANCHINI, E., GARCIA, C.C., PIMENTA, J.A. \& TOREZAN, J.M.D. 2010. Slope variation and population structure of tree species from different ecological groups in South Brazil. Anais da Academia Brasileira de Ciências 82:643-652.

BORGO, M., SILVA, S.M. \& PETEAN, M.P. 2002. Epífitos vasculares em um remanescente de floresta estacional semidecidual, município de Fênix, PR, Brasil. Acta Biologica Leopoldensia 24:121-130.

BOTREL, R.T., OLIVEIRA FILHO, A.T., RODRIGUES, L.A. \& CURI, N. 2002. Influência do solo sobre a composição florística e estrutura da comunidade arbóreoarbustiva de uma floresta estacional semidecidual em Ingaí, MG. Revista Brasileira de Botânica 25: 195-213.

CAMPOS, J.B. \& COSTA FILHO, L.V. 2006. Sistema ou conjunto de unidades de conservação? In Unidades de Conservação: Ações para valorização da biodiversidade (E. Schaitza, coord.). Instituto Ambiental do Paraná, Curitiba, p.17-22.

CARLIER, J.D., REIS, A., DUVAL, M.F., D’EECKENBRUGGE, C. \& LEITÃO, J.N. 2004. Genetic maps of RAPD, AFLP and 1RRS markers in Ananas bracteatus and A. comosus using the pseudo-testcross strategy. Plant Breeding 123: 186-192.

CARVALHO, J., MARQUES, M.C.M., RODERJAN, C.V., BARDDAL, M. \& SOUSA, S.G.A. 2009. Relação entre a distribuição das espécies de diferentes estratos e as características do solo de uma floresta aluvial no Estado do Paraná, Brasil. Acta Botanica Brasilica 23:1-9.

CARVALHO, D.A., OLIVEIRA FILHO, A.T., VILELA, E.A., CURI, N., VAN DEN BERG, E., FONTES, M.A.L. \& BOTEZELLI, L. 2005. Distribuição de espécies arbóreo-arbustivas ao longo de um gradiente de solos e topografia em um trecho de floresta ripária do Rio São Francisco em Três Marias, MG, Brasil. Revista Brasileira Botânica 28:28-345.
CAVIGLIONE, J.H., KIIHL, L.R.B., CARAMORI, P.H. \& OLIVEIRA, D. 2000. Cartas climáticas do Paraná. Instituto Agronômico do Paraná, Londrina.

COSTA, J.T., KRANZ, W., ESTEVAN, D., BIANCHINI, E. \& FONSECA, I.C.B. 2008. Ocorrência de espécies exóticas no Parque Florestal de Ibiporã. Cadernos da Biodiversidade 5:53-64.

DETTKE, G.A., ORFRINI,A.C. \& MILANESE-GUTIERRE, M.A. 2008. Composição florística e distribuição de epífitas vasculares em um remanescente alterado da Floresta Estacional Semidecidual do Paraná, Brasil. Rodriguésia 59:859-872.

DIAS, M.C., VIEIRA, A.O.S. \& PAIVA, M.R.C. 2002. Florística e fitossociologia das espécies arbóreas da bacia do rio Tibagi. In A Bacia do Rio Tibagi (M.E. Medri, E. Bianchini, O.A. Shibatta \& J.A. Pimenta, eds.). Londrina, p.109-124.

DURIGAN, G., BERNACCI, L.C., FRANCO, G.A.D.C., ALBOCZ, J.P.M. \& CATHARINO, E.L.M. 2008. Estádio sucessional e fatores geográficos como determinantes da similaridade florística entre comunidades florestais no Planalto Atlântico, estado de São Paulo, Brasil. Acta Botanica Brasilica 22:51-62.

DURIGON, J. \& WAECHTER, J.L. 2011. Floristic composition and biogeographic relations of a subtropical assemblage of climbing plants. Biodiversity and Conservation 20:1027-1044.

FIDALGO, O. \& BONONI, V.L.R. 1989. Técnicas de coleta, preservação e herborização de material botânico. Instituto de Botânica, São Paulo.

FUTUYMA, D.J. 1996. Ecology: the environental context of evolutionary changes. In Evolucionary biology (D.J. Futuyma, ed.). Sinauer, Sunderland, p.59-84.

GANDOLFI, S., LEITÃO FILHO, H.F. \& BEZERRA, C.L. 1995. Levantamento florístico e caráter sucessional das espécies arbustivo-arbóreas de uma floresta mesófila semidecídua no Município de Guarulhos, SP. Revista Brasileira de Biologia 55:753-767.

GUARATINI, M.T.G, GOMES, E.P.C., TAMASHIRO, J.Y. \& RODRIGUES, R.R. 2008. Composição florística da Reserva Municipal de Santa Genebra, Campinas, SP. Revista Brasileira de Botânica 31:323-337.

IAP - Instituto Ambiental do Paraná. 2009. Plano de Manejo do Parque Florestal de Ibiporã. http://www.uc.pr.gov. br/arquivos/File/Plano_de_Manejo/PE_Ibipora/Plano ManejoPEIbipora/pdf> (acesso em 04/01/2009).

IBAMA - Instituto Brasileiro do Meio Ambiente e Recursos Naturais. 1992. Portaria no $37-\mathrm{N}$, de 03 de abril de 1992. Lista de espécies da flora ameaçada de extinção. Disponível em: <http:www.ambientebrasil.com.br/ > (acesso em 19/03/2008).

INÁCIO, C.D. \& JARENKOW, J.A. 2008. Relações entre a estrutura da sinúsia herbácea terrícola e a cobertura do dossel em floresta estacional do sul no Brasil. Revista Brasileira de Botânica 31:41-51. 
KAGEYAMA, P.F. \& CASTRO, C.F.A. 1989. Sucessão secundária, estrutura genética e plantações de espécies arbóreas nativas. Instituto de Pesquisas e Estudos Florestais 41/42:83-93.

KINOSHITA, L.S., TORRES, R.B., FORNI-MARTINS, E.R., SPINELLI, T., AHN, Y.J. \& CONSTÂNCIO, S. 2006. Composição florística e síndrome de polinização e de dispersão da mata do Sítio São Francisco, Campinas, SP, Brasil. Acta Botanica Brasilica 20: 313-327.

KOTCHETKOFF-HENRIQUES, O., JOLY, C.A. \& BERNACCI, L.C. 2005. Relação entre solo e a composição florística do remanescente de vegetação natural no município de Ribeirão Preto, SP. Revista Brasileira de Botânica 28:541-562.

LEITÃO FILHO, H.F. 1987. Considerações sobre a florística de florestas tropicais e sub-tropicais do Brasil. Instituto de Pesquisas e Estudos Florestais 35:41-46.

MEIRA NETO, J.A.A., MARTINS, F.R. \& SOUZA, A.L. 2005. Influência da cobertura e do solo na composição florística do sub-bosque em uma floresta estacional semidecidual em Viçosa, MG, Brasil. Acta Botanica Brasilica 19:473-486.

MMA - Ministério do Meio Ambiente. 2000. Lei no 9.985, de 18 de julho de 2000. Sistema Nacional de Unidades de Conservação da Natureza - SNUC. Diário Oficial da República Federativa do Brasil, 19 jul. Brasília.

MORELLATO, L.P. \& LEITÃO FILHO, H.F. 1998. Levantamento florístico da comunidade de trepadeiras de uma floresta semidecídua no sudeste do Brasil. Boletim do Museu Nacional do Rio de Janeiro-Série Botânica 103:1-15.

MURCIA, C. 1995. Edge effects in fragmented forests: implications for conservation. Trends in Ecology and Evolution, 10:58-62.

NUNES, Y.R.F., MENDONÇA, A.V.R., BOTEZELLI, L., MACHADO, E.L.M. \& OLIVEIRA FILHO, A.T. 2003. Variações da fisionomia, diversidade e composição de guildas da comunidade arbórea em fragmento de floresta semidecidual de Lavras, MG. Acta Botanica Brasilica 17:213-229.

OLIVEIRA FILHO, A.T., CARVALHO, D.A., VILELA, E.A., CURI, N. \& FONTES, M.A.L. 2004. Diversity and structure of the tree community of a fragment of tropical secondary Forest of the Brazilian Atlantic Forest domain 15 and 40 years after logging. Revista Brasileira Botânica 27:85-701.
PAULA, C.C., SARTIRI, M.A. \& GUARÇONI, E.A.E. 2008. Bromeliaceae Juss. of the Municipal Park of Itajuru, Muriaé, Minas Gerais, Brazil. Selbyana 29:128-130.

RESENDE, A.A. \& RANGA, N.T. 2005. Lianas da estação ecológica do Noroeste Paulista, São José do Rio Preto, Mirassol, SP, Brasil. Acta Botanica Brasilica 19: 273-279.

RIZZINI, C.T. 1997. Tratado de fitogeografia do Brasil: aspectos ecológicos, sociológicos e florísticos. Âmbito Cultural, Rio de Janeiro.

RODERJAN, C.V. \& MEDEIROS, M.L.C.M. 1980. Caracterização da vegetação do Horto Florestal de Ibiporã. Instituto Ambiental do Paraná, Curitiba.

RODERJAN, C.V., GALVÃO, F., KUNIYOSHI, Y.S. \& HATSCHBACH, G.G. 2002. As unidades fitogeográficas do Estado do Paraná. Ciência \& Ambiente 24:75-92.

SCHEINVAR, L. 1985. Cactáceas. In Flora Ilustrada Catarinense (R. Reitz, ed.). Herbário Barbosa Rodrigues, Itajaí.

SILVA, F.C. \& SOARES-SILVA, L.H. 2000. Arboreal flora of the Godoy Forest State Park, Londrina, PR, Brazil. Edinburgh Journal of Botany 57:107-120.

SOARES-SILVA, L.H. \& BARROSO, G.M. 1992. Fitossociologia do estrato arbóreo da floresta na porção norte do Parque Estadual Mata dos Godoy, Londrina, PR, Brasil. In Anais do VIII Congresso da Sociedade Botânica de São Paulo. Sociedade Botânica de Sâo Paulo, Campinas, p.101-112.

STRANGUETTI, V. \& RANGA, N.T. 1998. Levantamento florístico das espécies vasculares da floresta estacional mesófila Semidecidual da Estação Ecológica de Paulo de Faria - SP. Revista Brasileira de Botânica 21: 289-298.

TOREZAN, J.M. \& SILVEIRA, M. 2002. Fatores ambientais, diversidade e similaridade em florestas da bacia do rio Tibagi. In: A Bacia do Rio Tibagi (M.E. Medri, E. Bianchini, O.A. Shibatta \& J.A. Pimenta, eds.). Londrina, p.125-129.

TRABAQUINI, K., MIGLIORANZA, E., FRANÇA, V. \& VIEIRA, A.O. 2007. Análise espacial de fragmentos florestais com ocorrência de Jaracatiá no norte do Paraná - Brasil. RA’ E GA 14:193-203.

VELOSO, H.P., RANGEL FILHO, A.L.R. \& LIMA, J.C.A. 1991. Classificação da vegetação brasileira, adaptada a um sistema universal. Instituto Brasileiro de Geografia e Estatística, Rio de Janeiro. 